Pacific Journal of 


\title{
ANALYTIC SUBGROUPS OF AFFINE ALGEBRAIC GROUPS, II
}

\begin{abstract}
ANDY R. MAGID
Dedicated to Gerhard Hochschild on the occasion of his 65th birthday

Let $H$ be Zariski-dense analytic subgroup of the connected linear complex algebraic group $G$. It is known that there is a torus $T$ in $G$ with $G=H T$ and $H \cap T$ discrete in $H$. This paper gives equivalent conditions for $H \cap T$ to be trivial, and considers the connection between these conditions and left algebraic group structures on $H$ induced from the coordinate ring of $G$.
\end{abstract}

Let $G$ be a connected linear complex algebraic group, and let $H$ be a Zariski-dense analytic subgroup of $G$ which is integral in the sense of [2, Defn. 1, p. 386]. In [10, Thm. 3] it was shown that there exists an algebraic torus $T$ in $G$ with $G=H T$ such that the Lie algebra of $T$ is a vector space complement to the Lie algebra of $H$ in the Lie algebra of $G$; $T$ is called a complementary torus to $H$ in $G$. The principal results of this paper deal with conditions under which such a complementary torus meets $H$ trivially. The existence of such a torus is connected, by [10, Prop. 6] and [10, Prop. 7], to left algebraic group structures on $H$ in the sense of [8, Defn. 2.1].

We recall some terminology: let $H$ be an analytic group, let $f$ be an analytic function on $H$, and let $x$ be in $H$. Then $x \cdot f$ (respectively, $f \cdot x)$ is the function on $H$ whose value at $y$ is $f(y x)$ (respectively, $f(x y)) . \quad f$ is representative if $\{x \cdot f \mid x \in H\}$ spans a finite-dimensional vector space, and $R(H)$ denotes the Hopf algebra of all representative functions on $H$ [5]. A representative function $f$ on $H$ is semi-simple if the representation of $H$ on the span of $\{x \cdot f \mid x \in H\}$ is semi-simple, and $R(H)_{s}$ denotes the subalgebra of all semi-simple representative functions on $H$ [5]. An analytic left algebraic group structure on $H$ is a finite-type $C$-subalgebra $A$ on $R(H)$ such that (1) if $f \in A$ and $x \in H$, $f \cdot x \in A$, and (2) evaluations at element of $H$ correspond bijectively to $C$-algebra maps from $A$ to $C$ [8, Defn. 2.1]. A nucleus of $H$ is a closed, solvable, simply connected normal subgroup $K$ such that $H / K$ is reductive [6, p. 112]. An additive character of $H$ is a homomorphism from $H$ to the additive analytic group $C$ and $X^{+}(H)$ is the free abelian group of additive characters of $H . H$ is an $F R$ group if $H$ has a faithful finite-dimensional representation; if $V$ is the space of such a representation then $H$ is a Zariski-dense analytic subgroup of the Zariski-closure of $H$ in $\mathrm{GL}(V)$ which is an algebraic group. 
$L(H)$ is the Lie algebra of $H$. If $G$ is a complex algebraic group, $k[G]$ denotes the affine coordinate ring of $G$, and we refer to the complex topology on $G$ as the strong topology.

Let $H$ be a connected, commutative $F R$ analytic group. By [10, Lemma 1], $H=V \times T$, where $V$ is a complex vector group and $T$ is a multiplicative torus. Then $T$ is the intersection of the kernels of the additive characters of $H$, so $T$ is the unique maximal torus of $H$.

LEMMa 1. Let $H$ be a connected commutative $F R$ analytic group and let $T$ be the unique maximal torus of $H$.

(1) A closed analytic subgroup $U$ of $H$ is a nucleus if and only if $H=U T$ with $U \cap T=\{e\}$.

(2) There is a one-one correspondence between the nuclei of $H$ and the vector space complements to $L(T)$ in $L(H)$.

Proof. Write $H=V \times T$ with $V$ a complex vector group, and let $p_{1}: H \rightarrow V$ and $p_{2}: H \rightarrow T$ be the projections. Let $U$ be a nucleus of $H$. Then $p_{1}(U)$ is a vector subgroup of $V$. If $p_{1}(U) \neq V$, there is a nonzero linear functional $p$ on $V$ with $p\left(p_{1} U\right)=0$. Let $q=p p_{1}$. Then $q$ is an additive character of $H$ with $q(U)=0$, so $q$ induces an onto additive character of $H / U$. Since $H / U$ is reductive and commutative, $H / U$ is a torus and hence has no surjective additive characters. Thus $p_{1}(U)=V$ and hence $T \rightarrow H / U$ is onto. Then there is a subtorus $T_{1}$ of $T$ such that $L\left(T_{1}\right) \rightarrow L(H / U)$ is an isomorphism. Since $T_{1}$ and $H / U$ are tori, $T_{1} \rightarrow H / U$ is algebraic, and hence an isogeny. In particular, $T_{1} \cap U$ is finite. But $U$ is simply connected, and hence a vector group, so $T_{1} \cap U=\{e\}$. Then $H=T_{1} \times U$, so $T_{1}$ is a maximal torus of $H$, so $T=T_{1}$. This establishes half of (1). For the other half, if $H=U T$ with $U \cap T=\{e\}$, then $L(H)=$ $L(U) \oplus L(T)$. Since $\operatorname{Ker}\left(\exp _{H}\right)$ is contained in $T$, $\exp _{H}: L(U) \rightarrow U$ is an isomorphism, so $U$ is simply connected. $U$ is solvable and normal, and $H / U$ is isomorphic to $T$, and hence reductive, so $U$ is a nucleus of $H$, and (1) obtains.

For (2), if $U$ is a nucleus of $H$ then (1) implies that $L(H)=$ $L(U) \oplus L(T)$. Conversely, if $L(H)=M \oplus L(T)$, let $U=\exp _{H}(M)$. Then $\exp _{I I}^{-1}(U)=M+\operatorname{Ker}\left(\exp _{H}\right)$ is closed in $L(H)$, so $U$ is a closed analytic subgroup of $H$. Also, $U T=H$ since $L(H)=M \oplus L(T)$ and $M=L(U)$. If $x \in U \cap T$, there are $m \in M$ and $y \in L(T)$ with $\exp _{H}(m)=$ $\exp _{I I}(y)=x$. Then $m-y \in \operatorname{Ker}\left(\exp _{I I}\right) \subseteq L(T)$ so $m \in L(T) \cap M=0$ and $x=e$. Thus $U \cap T=\{e\}$ and by (1), $U$ is a nucleus of $H$.

Lemma 2. Let $H$ be a connected analytic group, let $R$ be the radical of $H$, and let $K$ be a nucleus of $H$. 
(1) $(H, R) \subseteq K \leqq R$ and $K$ is a nucleus of $R$.

(2) If $L$ is a closed simply connected normal subgroup of $H$ with $L \cong K$, then $K / L$ is a nucleus of $H / L$.

Proof. (1) $K$ is contained in $R$ since $K$ is a connected closed solvable normal subgroup of $H$. Let $f: H \rightarrow H / K$ denote the projection. Then $f(R)$ is the radical of the reductive group $H / K$, so $e=(H / K$, $f(R))=f(H, R)$ and $(H, R)$ is contained in $K$. Also, $K$ is a closed, simply connected solvable normal subgroup of $R$, and $R / K$ is the radical of $H / K$. Since $H / K$ is reductive, its radical is a torus so $K$ is a nucleus of $R$. (2) $K / L$ is a closed, simply connected solvable normal subgroup of $H / L$ and $H / L / K / L=H / K$ is reductive, so $K / L$ is a nucleus of $H / L$.

The preceding lemmas combine with [10, Thm. 10] to yield the following characterization of nuclei.

THEOREM 3. Let $H$ be a connected FR analytic group, let $R$ be the radical of $H$, let $\bar{R}=R /(H, R)$, let $T$ be the unique maximal torus of $\bar{R}$, and let $f: R \rightarrow \bar{R}$ be the canonical map. Then the nucle $i$ of $H$ are the groups $f^{-1}(U)$, where $U$ is a closed analytic subgroup of $\bar{R}$ with $\bar{R}=U T$ and $U \cap T=\{e\}$.

Proof. [10, Thm. 10] shows that $(H, R)$ is closed in $H$ and that every $f^{-1}(U)$ is a nucleus. Conversely, if $K$ is a nucleus of $H$ then by Lemma 2, part (1), $(H, R) \subseteq K \subseteq R$ and $K$ is a nucleus of $R$. By Lemma 2, part (2), with $L=(H, R) \subseteq R, K /(H, R)$ is a nucleus of $\bar{R}$, and by Lemma $1, K /(H, R) \times T=H$, so $K=f^{-1}(K /(H, R))$ is of the desired form.

Theorem 3 allows us to improve [10, Thm. 10] somewhat:

CoRollary 4. Let $G$ be a connected linear algebraic group, $H$ a Zariski-dense analytic subgroup of $G$ and $K$ a nucleus of $H$. Then there is a reductive subgroup $Q$ of $H$ Zariski-closed in $G$ and a complementary torus $T$ of $H$ in $G$ such that $H=K Q$ with $K \cap Q=\{e\}$ and $(T, Q)=\{e\}$.

Proof. [10, Thm. 10] establishes the existence of $T$ and $Q$ when $K$ has the form $f^{-1}(U)$ as in Theorem 3, and Theorem 3 shows that $K$ always has this form.

Also, Theorem 3 and Lemma 1 show that, in the notation of Theorem 3, the set of nuclei of $H$ corresponds bijectively to the set 
of vector space complements to $L(T)$ in $L(\bar{R})$. We now show that this latter set carries the structure of a complex vector space.

To simplify notation, let $V$ be a finite-dimensional complex vector space and let $W$ be a subspace of $V$. Let $S$ be the set of vector space complements to $W$ in $V$. Fix $M_{0}$ in $S$, and let $M$ be any element of $S$. Let $p: V \rightarrow M_{0}$ and $q: V \rightarrow W$ be the projections. Since $\operatorname{Ker}(p)=$ $W$ and $W \cap M=0, \operatorname{Ker}(p \mid M)=0$. Since $\operatorname{dim} M=\operatorname{dim} V-\operatorname{dim} W=$ $\operatorname{dim} M_{0}, p \mid M$ is an isomorphism. Let $f_{M}=q \circ(p \mid M)^{-1}: M_{0} \rightarrow W$. Then $M=\left\{m+f_{M}(m) \mid m \in M_{0}\right\}$, and $M \rightarrow f_{M}$ is a bijection between $S$ and $\operatorname{Hom}_{C}\left(M_{0}, W\right)$. Thus $S$ carries the structure of a complex vector space.

We relate this calculation to sets of nuclei:

THEOREM 5. Let $H$ be a connected $F R$ analytic group, and let $R$ be the radical of $H$. Then the set of nuclei of $H$ is a complex vector space of dimension $r d-r_{1} d-d^{2}$, where $r=\operatorname{dim}(L(R)), r_{1}=$ $\operatorname{dim} L((H, R))$ and $d=\operatorname{rank}\left(X^{+}(H)\right)$.

Proof. Let $\bar{R}=R /(H, R)$ and let $T$ be the maximal torus of $\bar{R}$. As noted above, the set of nuclei corresponds bijectively to the set of vector space complements to $L(T)$ in $L(\bar{R})$ by Theorem 3 and Lemma 1. Let $U$ be a vector subgroup of $\bar{R}$ with $\bar{R}=U \times T$. By the above considerations, the set of vector space complements to $L(T)$ in $L(\bar{R})$ is in bijection with $\operatorname{Hom}_{c}(L(U), L(T))$. Let $\bar{H}=H /(H, R)$. Then $\bar{H}$ is also $F R$ (since $(H, R)$ is normal and Zariski-closed in any linear algebraic group in which $H$ is a Zariski-dense analytic subgroup), and $\bar{H}=\bar{R} S$ where $S$ is semi-simple since $\bar{R}$ is the radical of $\bar{H}$. Also, $\bar{R}$ is central in $\bar{H}$, so that $\bar{R} \cap S$ is central in $S$, and since $S$ is semi-simple and $F R$, the center of $S$ is finite. Thus $\bar{R} \cap S$ is finite. But every element of finite order of $\bar{R}$ lies in $T$, so $\bar{R} \cap S \subseteq T$ and $\bar{H}=U \times(T S)$. Now $X^{+}(H)=X^{+}(\bar{H})$, and since $T S$ is reductive, $X^{+}(\bar{H})=X^{+}(U)$. Thus $\operatorname{dim}(U)=\operatorname{dim}(L(U))=\operatorname{rank}\left(X^{+}(H)\right)=d$. Also $\operatorname{dim}(L(T))=\operatorname{dim}(L(\bar{R}))-\operatorname{dim}(L(U))=\operatorname{dim}(L(R))-\operatorname{dim}(L(H, R))-$ $\operatorname{dim} L(U)=r-r_{1}-d$. Thus $\operatorname{dim}\left(\operatorname{Hom}_{c}(L(U), L(T))=\left(r-r_{1}-d\right) d\right.$.

A similar description of the set of nuclei as a vector space was obtained by other means in [9, Cor. 2.2].

We now consider some further implications of Corollary 4. Let $G$ be a connected linear algebraic group, $H$ a Zariski-dense analytic subgroup, $K$ a nucleus of $H, Q$ a reductive subgroup of $G$, and $T$ a complementary torus of $H$ in $G$ such that $H=K Q$ with $K \cap Q=\{e\}$, and $(T, Q)=\{e\}$, as in the corollary. Then $P=T Q$ is a reductive subgroup of $G$ with $(P, P)=(Q, Q)$. We show now that $P$ contains a complementary torus $T^{\prime \prime}$ of $H$ in $G$ with $T^{\prime \prime} \cap Q=\{e\}$. 
Proposition 6. Let $P$ be a reductive algebraic group and let $Q$ be a reductive algebraic subgroup with $(P, P)=(Q, Q)$. Then there is an algebraic torus $T^{\prime \prime}$ in $P$ such that $P=Q T^{\prime \prime}$ with $Q \cap T^{\prime \prime}=\{e\}$.

Proof. Let $S=(Q, Q)=(P, P)$. We note that $S$ and $Q$ are normal in $P$. Let $R_{0}$ be the radical of $P$ and $R_{1}$ be the radical of $Q$, so $R_{1}$ is contained in $R_{0}$. Let $T_{1}$ be a torus in $S$ containing the center of $S$ [1, Cor. 11.1, p. 270]. Then $T_{0}=R_{0} T_{1}$ is a torus in $P$ and $T_{1} \leqq T_{0} \cap S$. We claim that $T_{0} \cap S=T_{1}$ : for if $r t \in T_{0} \cap S$ with $r \in R_{0}$ and $t \in T_{1}$, then $r \in R_{0} \cap S$ which is central in $S$ so $r \in T_{1}$ and $r t \in T_{1}$. Let $T_{0}^{\prime}=R_{1} T_{1}$. Then $T_{1} \subseteq T_{0}^{\prime} \cap S \subseteq T_{0} \cap S=T_{1}$, so $T_{0}^{\prime} \cap S=$ $T_{1}$. Also, $T_{1}$ is a subtorus of $T_{0}^{\prime}$, so by [1, Cor., p. 206] there is a torus $T^{\prime}$ in $T_{0}^{\prime}$ so that $T_{0}^{\prime}=T_{1} T^{\prime}$ and $T^{\prime} \cap T_{1}=\{e\}$. Since $T^{\prime} \cap S \subseteq$ $T_{0}^{\prime} \cap S=T_{1}, T^{\prime} \cap S=\{e\}$. Since $T_{0}^{\prime}$ is a subtorus of $T_{0}$, by [1, Cor., p. 206] again there is a torus $T^{\prime \prime}$ in $T_{0}$ so that $T_{0}=T_{0}^{\prime} T^{\prime \prime}$ and $T_{0}^{\prime} \cap$ $T^{\prime \prime}=\{e\}$. Since $T^{\prime \prime} \cap S \subseteq T_{0} \cap S=T_{1}$ and $T_{1} \subseteq T_{0}^{\prime}$ so $T^{\prime \prime} \cap T_{1}=\{e\}$, then $T^{\prime \prime} \cap S=\{e\}$. Let $T=T^{\prime \prime} T^{\prime \prime}$. Then $T_{0}=T_{1} T$ and $T_{1} \cap T=\{e\}$. Let $x=t_{1} t_{2}$ be in $T \cap S$ with $t_{1} \in T^{\prime}$ and $t_{2} \in T^{\prime \prime}$. Then $x \in T_{0} \cap S=T_{1}$ so $x \in T \cap T_{1}=\{e\}$ and $t_{1}=t_{2}^{-1}$. Since $T^{\prime} \cap T^{\prime \prime} \subseteq T_{0}^{\prime} \cap T^{\prime \prime}=\{e\}, t_{1}=e$. Then $T \cap S=\{e\}$. Now $Q=R_{1} S$ so $Q=T_{0}^{\prime} S$, and $T_{0}^{\prime}=T^{\prime} T_{1}$ with $T_{1} \subseteq S$, so $Q=T^{\prime} S$. Similarly, since $P=R_{0} S, P=T_{0} S$ and $T_{0}=$ $T_{0}^{1} T^{\prime \prime}=T_{1} T^{\prime} T^{\prime \prime}=T_{1} T$ with $T_{1} \subseteq S, P=T S$. Since $T=T^{\prime \prime} T^{\prime}, P=$ $T^{\prime \prime}\left(T^{\prime} S\right)=T^{\prime \prime} Q$. Now let $x \in T^{\prime \prime} \cap Q$. Since $x \in Q$ and $Q=T^{\prime} S, x=t s$ with $t \in T^{\prime}$ and $s \in S$. Then $s=t^{-1} x$ is in $T^{\prime \prime} T^{\prime \prime}=T$ and in $S$, and weshowed above that $T \cap S=\{e\}$. Thus $s=e$ and $x=t$ is in $T^{\prime \prime}$. But $x$ is also in $T^{\prime \prime}$ and $T^{\prime \prime} \cap T^{\prime}=\{e\}$ so $x=\{e\}$. Thus $Q \cap T^{\prime \prime}=\{e\}$, and the proposition follows.

CoRollary 7. Let $G$ be a connected linear algebraic group, $H$ a Zariski-dense analytic subgroup of $G$ and $K$ a nucleus of $H$. Then there is a reductive subgroup $Q$ of $H$ Zariski-closed in $G$ and a complementary torus $T^{\prime \prime}$ to $H$ in $G$ such that $H=K Q$ with $K \cap Q=$ $\{e\}, T^{\prime \prime} \cap Q=\{e\}$, and $T^{\prime \prime}$ normalizes $Q$.

Proof. Let $Q$ and $T$ be as in Corollary 4. Let $P=T Q$. Then $P$ is reductive and $(P, P)=(Q, Q)$. Let $T^{\prime \prime}$ be as in Proposition 6 . Then $G=H T=K Q T=K P=K Q T^{\prime \prime}=H T^{\prime \prime}$, and $L(G)=L(H) \oplus$ $L(T)=L(K) \oplus L(Q) \oplus L(T)=L(K) \oplus L(P)=L(K) \oplus L(Q) \oplus L\left(T^{\prime \prime}\right)=$ $L(H) \oplus L\left(T^{\prime \prime}\right)$, so $T^{\prime \prime}$ is a complementary torus to $H$ in $G$, and $Q$ and $T^{\prime \prime}$ possess the desired properties.

The examples following [10, Thm. 3] show that, in the notation of Corollary 7, it is not always possible to find a $T^{\prime \prime}$ with $T^{\prime \prime} \cap H=\{e\}$. Complementary tori with this property are connected to left algebraic 
group structures on $H$ [10, Prop. 6], and we now examine when such exist.

We will need to use some facts about representation theory in this examination. We fix the following terminology: if $H$ is an analytic group, an $H$-module $V$ is a finite-dimensional complex vector space with an analytic left $H$-action; we let $r_{\mathrm{r}}: H \rightarrow \mathrm{GL}(V)$ be the corresponding representation. The associated semi-simple module to $V$ is the direct sum $V^{\prime}$ of the $H$-module composition factors of $H$; we let $r_{V}^{\prime}=r_{r}$, and call $r_{v}^{\prime}$ the associated semi-simple representation. If $H$ is an analytic subgroup of the analytic group $G$, then every $G$-module is, by restriction, an $H$-module. In this case, if $V$ is an $H$-module, we say that $r_{V}$ extends to $G$ if there is a $G$-module $W$ containing $V$ as an $H$-submodule. In [3], a criterion is given for determining when a representation of $H$ extends to $G$ in the case $H$ is a normal semi-direct factor of $G$.

THEOREM 8. Let $G$ be a connected linear algebraic group and $H$ a Zariski-dense analytic subgroup of $G$. Then the following are equivalent:

(1) Every additive character of $H$ is the restriction of an additive character of $G$.

(2) There is a complementary torus $T$ to $H$ in $G$ with $T \cap H$ finite.

(3) There is a complementary torus $T^{\prime \prime}$ to $H$ in $G$ with $T^{\prime \prime} \cap$ $H=\{e\}$.

(4) Every nucleus of $H$ is a nucleus of $G$.

(5) Every $H$-module is an $H$-submodule of a $G$-module.

Proof. (1) and (2) are equivalent by [10, Thm. 3] and (3) implies (2) trivially. We next show that (2) implies (4). Let $K$ be a nucleus of $H$ and let $T, Q$ be as in Corollary 4. Let $P=T Q$. Then $P$ is a reductive subgroup of $G$ and $G=K P$. We assume $T \cap H$ is finite. By [10, Thm. 3], $H$ is strongly closed in $G$, and hence $K$ is a strongly closed simply connected analytic subgroup of $G . K$ is normal in $G$ since $H$ is Zariski-dense in $G$ and $K$ is normal in $H$. $K \cap P$ is solvable and normal in $P$, so $K \cap P$ is contained in the center $Z$ of the reductive group $P . \quad Z=T Z^{\prime}$, where $Z^{\prime}$ is the center of $Q$. Let $x \in K \cap P$. Then $x=t q$ where $t \in T$ and $q \in Z^{\prime}$. Since $x \in H$ and $q \in H, t \in T \cap H$. Let $n$ be the order of $T \cap H$. Then $x^{n}=q^{n}$, so $q^{n} \in K \cap Q=\{e\}$. Let ${ }_{n} Z^{\prime}$ denote the $n$-torsion in $Z^{\prime}$. Then ${ }_{n} Z^{\prime}$ is finite since $Q$ is reductive, and $K \cap P \subseteq(T \cap H)\left({ }_{n} Z^{\prime}\right)$ so $K \cap P$ is finite. Since $K$ is simply connected, $K \cap P=\{e\}$. Thus $G / K=P$ is reductive, so $K$ is a nucleus of $G$ and (2) implies (4). We now show that (4) implies (3). Let $K, T, Q, P$ be as above. Since $K$ is then 
a nucleus of $G, G / K=P / K \cap P$ is reductive. The analytic map $f: P \rightarrow P / K \cap P$ is then a morphism of algebraic groups by [10, Lemma A1]. Since $f$ induces an isomorphism on Lie algebras, it follows that $f$ has finite kernel, i.e., $P \cap K$ is finite, and since $K$ is simply connected, $P \cap K=\{e\}$. Thus $G=K P$ with $P \cap K=\{e\} . \quad$ By Proposition 6, $P=Q T^{\prime \prime}$ with $T^{\prime \prime} \cap Q=\{e\}$. It follows that $G=$ $K Q T^{\prime \prime}=H T^{\prime \prime}$ and $T^{\prime \prime} \cap H=\{e\}$, so (4) implies (3). We next show that (3) implies (5): Assume condition (3) holds; i.e., $G=H T$ with $T$ a torus in $G$ with $T \cap H=\{e\}$. Let $V$ be an $H$-module and let $r=r_{r}$. be the corresponding representation. Let $R$ be the radical of $H$. By 13, Thm. 2.2, p. 215], $V$ is an $H$-submodule of a $G$-module if and only if $r^{\prime}((G, R))=1$. We claim that $(G, R)=(H, R)$. First, $(H, R)$ is contained in the unipotent radical of $G$, so $(H, R)$ is Zariski-closed in $G$. Let ()$_{c}$ denote Zariski-closure. Then $(H, R)_{c}=\left(H_{c}, R_{c}\right)$ by [1, Prop., p. 108]. Thus $(G, R)=\left(H_{c}, R\right) \leqq\left(H_{c}, R_{c}\right)=(H, R)_{c}=(H, R)$ and it follows that $(G, R)=(H, R)$. Since $(H, R)$ acts trivially on simple $H$-modules, $r^{\prime}((G, R))=r^{\prime}((H, R))=1$, so every $H$-module is an $H$-submodule of a $G$-module. Finally, we show that (5) implies (1): Let $f \in X^{+}(H), f \neq 0$ and let $V$ be the two dimension complex space with basis $e_{1}, e_{2}$ and let $H$ act on $V$ by $h e_{1}=e_{1}$ and $h e_{2}=$ $f(h) e_{1}+e_{2}$ for $h \in H$. Then $V$ is an $H$-module. Let $W_{0}$ be a $G$-module containing $V$ as an $H$-submodule. Let $K$ be the kernel of $f$. Since $(G, G)=(H, H)$ is contained in $K, K$ is normal in $G$. Let $W=$ $\left\{x \in W_{0} \mid k x=x\right.$ for all $k$ in $\left.K\right\}$. Since $K$ is normal in $G, W$ is a $G$ submodule of $W_{0}$ and $W$ contains $V$. Let $\bar{H}=r_{w}(H)$ and $\bar{G}=r_{w}(G)$. $W$ is a $\bar{G}$ - and $\bar{H}$-module, and since $K \cong \operatorname{Ker}\left(r_{W}\right)$ and $(G, G) \leqq K, \bar{G}$ is abelian. Let $T$ be the unique maximal torus of $\bar{G}$. If every additive character of $\bar{G}$ vanishes on $\bar{H}$, then $\bar{H}$ is contained in $T$. $W$ is semi-simple as a $T$-module, so $W$ is semi-simple as an $\bar{H}$-module, if $\bar{H} \leqq T$. But then $V$ is also semi-simple as an $\bar{H}$-module, hence as an $H$-module, so $f=0$, contrary to assumption. Thus there is an additive character of $\bar{G}$ which is not trivial on $\bar{H}$. This character defines an additive character $g$ on $G$ whose kernel contains $K$ but whose restriction to $H$ is not trivial. Let $g_{1}$ be the restriction of $g$ to $H$. Both $g_{1}$ and $f$ induce isomorphisms $H / K \rightarrow C$, so there is a nonzero scalar $\alpha$ such that $\alpha g_{1}=f$. It follows that $f$ is the restriction of $a g$ to $H$, and $a g \in X^{+}(G)$. Thus (5) implies (1), and Theorem 8 is complete.

Condition (3) of Theorem 8 is related to the existence of analytic left algebraic group structures on $H$ by [10, Prop. 6] and [10, Prop. 71. Thus the other conditions, especially condition (1), are also so related, as the following corollary makes precise.

Corollary 9. Let $H$ be an FR analytic group, and $B$ a Hopf- 
subalgebra of $R(H)$ a finite type over $C$. Then the following are equivalent:

(1) $B$ contains an analytic left algebraic group structure on $H$.

(2) $B$ separates the points of $H$ and contains $X^{+}(H)$.

Proof. Assume (1) and let $A$ be the left algebraic group structure. Let $B^{\prime}$ be the smallest sub-Hopf-algebra of $R(H)$ containing $A$, and let $G^{\prime}$ be the algebraic group with $k\left[G^{\prime}\right]=B^{\prime}$. By [10, Prop. 7], $H$ is a Zariski-dense and strongly closed analytic subgroup of $G^{\prime}$ and there is a complementary torus $T^{\prime \prime}$ to $H$ in $G^{\prime}$ with $T^{\prime} \cap H=\{e\}$. By Theorem 8, every additive character of $H$ extends to $G^{\prime}$. Since additive analytic characters of algebraic groups are algebraic, the additive characters are in $k\left[G^{\prime}\right]=B^{\prime}$. Thus $X^{+}(H) \subseteq B^{\prime} \leqq B$. By definition, $A$ separates points of $H$, hence so does $B$, so (1) implies (2). Conversely, assume (2). Let $G$ be the algebraic group with $k[G]=B$. Then $H$ becomes a Zariski-dense analytic subgroup of $G$. Let $f \in X^{+}(H)$. Then $f$ is a primitive element of $R(H)$ : i.e., the comultiplication sends $f$ to $f \otimes 1+1 \otimes f$, so $f$ is primitive in $B$ and hence defines an additive character of $G$. By Theorem 8, there is a complementary torus $T$ to $H$ in $G$ with $T^{\prime \prime} \cap H=\{e\}$. By [10, Prop. 6], $A=B^{T}$ is an analytic left algebraic group structure on $H$ and $A$ is contained in $B$ so (2) implies (1).

Let $H$ be an analytic group and $A$ a subgroup of $R(H)$. We recall that $A_{s}=A \cap R(H)_{s}$ denotes the semi-simple representative functions in $A$. If $A$ is a left algebraic group structure on $H, A$ is said to be normal basic if for every $f$ in $A_{s}$ and $x$ in $H, x \cdot f$ and $f \cdot x$ are in $A_{s}$ [6, p. 116], and a sub-Hopf-algebra of $R(H)$ of finite type over $C$ is regular if it contains a normal basic left algebraic group structure on $H[7, \mathrm{p} .873]$. We will now interpret this concept in terms of complementary tori. The following lemma determines the semi-simple part of the coordinate ring of an algebraic group.

Lemma 10. Let $G$ be a connected linear complex algebraic group and let $U$ be its unipotent radical. Then $k[G]_{s}=k[G]^{U}$.

Proof. $k[G]^{U}=k[G / U]$ and since $G / U$ is reductive, $k[G / U]_{s}=$ $k[G / U]$. Thus $k[G]^{U}$ is contained in $k[G]_{s}$. Conversely, let $f \in k[G]_{s}$, let $V=\langle x \cdot f \mid x \in G\rangle$ and let $r=r_{V}$ be the associated representation. Since $V$ is semi-simple, $U$ is in the kernel of $r$. Since $f \in V, x \cdot f=$ $r(x) f=f$ for all $x$ in $U$, so $f$ is in $k[G]^{U}$. Thus $k[G]_{s}$ is contained in $k[G]^{U}$ and the result follows. 
THEOREM 11. Let $G$ be a connected linear algebraic group and $H$ a Zariski-dense analytic subgroup of $G$. Let $Q$ be a maximal reductive subgroup of $H$. Then the following conditions are equivalent:

(1) Every additive character of $H$ is the restriction of an additive character of $G$, and there is a normal algebraic subgroup $L$ of $G$ such that $L Q=G$ and $L \cap Q=\{e\}$.

(2) There is a complementary torus $T$ to $H$ in $G$ with $T \cap H=$ $\{e\}$ and $(T, Q)=\{e\}$.

(3) $k[G]^{T}$ is a normal basic left algebraic group structure on $H$ for some complementary torus $T$ to $H$ in $G$.

(4) $k[G]$ is a regular sub-Hopf-algebra of $R(H)$.

Proof. Assume condition (1) and let $g: G \rightarrow G$ be the algebraic endomorphism with $\operatorname{Ker}(g)=L$ and $g(x)=x$ for all $x$ in $Q$. Let $K=L \cap H$. Then $K$ is the kernel of the restriction of $g$ to $H$, and $H=K Q$ with $K \cap Q=\{e\}$, so $K$ is a connected closed normal subgroup of $H$. By [10, Thm. 10], $K=K_{0} Q_{0}$ where $K_{0}$ is a nucleus of $K$ and $Q_{0}$ is a reductive subgroup of $K$ with $Q_{0} \cap K_{0}=\{e\}$. Since $Q$ is maximal reductive in $H$, some conjugate of $Q_{0}$ is contained in $Q$ : then there is an $x \in H$ with $x Q_{0} x^{-1} \subseteq Q$. But $x Q_{0} x^{-1} \subseteq K$ so $Q_{0}=\{e\}$ and $K=K_{0}$. Thus $K$ is simply connected and hence a nucleus of $H$. Let $\bar{K}$ be the Zariski-closure of $K$ in $G$. Then $\bar{K} \leqq L$, and $\bar{K} Q$ is Zariski-closed in $G$. Since $H \subseteq \bar{K} Q$, and $H$ is Zariski-dense in $\bar{K} Q=G$, it follows that $\bar{K}=L$. In particular, $L$ is solvable. Since every additive character of $H$ extends to $G$, Theorem 8 implies that $K$ is a nucleus of $G$. Let $P$ be a (necessarily maximal) reductive subgroup of $G$ such that $G=K P$ with $K \cap P=\{e\}$. If necessary, we replace $P$ by a conjugate so that $Q \subseteq P$. Let $T=L \cap P$. Then $P=T Q$ with $T \cap Q=\{e\}$, and $T$ is a closed connected normal algebraic subgroup of $P$ which is solvable since $L$ is solvable. It follows that $T$ is a torus with $(T, Q)=\{e\}$, and $G=K P=K T Q=H T$ with $T \cap H \subseteq K \cap P=\{e\}$. Thus condition (2) obtains.

Now assume $T$ is as in condition (2). By [10, Prop. 6], $A=$ $k[G]^{T}$ is an analytic left algebraic group structure on $H$. We need to show if $f \in A_{s}$ and $x \in H$, then $x \cdot f$ and $f \cdot x$ are in $A_{s}$. Let $U$ be the unipotent radical of $G$ and let $L=U T$. By Lemma 10, $A_{s}=$ $k[G]^{L}$. Let $K$ be a nucleus of $H$. Then $G=H T=K Q T$ and it follows that $Q T$ is a maximal reductive subgroup of $G$. By [4, Thm. 14.2, p. 96], $G=U Q T=L Q$, and $Q$ normalizes $U$ and $T$ so $L$ is normal in $G$. Thus if $f \in k[G]^{L}$ and $x \in G, x \cdot f$ and $f \cdot x$ are in $k[G]^{L}$. So condition (3) obtains.

Condition (3) implies condition (4) by definition, and condition (4) implies condition (1) by [7, Thm. 2.1, p. 875]. 


\section{REFERENCES}

1. A. Borel, Linear Algebraic Groups, W. A. Benjamin, Inc., New York, 1969.

2. N. Bourbaki, Lie Algebras and Lie Groups, Part 1, Addison-Wesley, Reading, Mass., 1975.

3. G. Hochschild, The Structure of Lie Groups, Holden-Day, San Francisco, 1965.

4. - Introduction to Affine Algebraic Groups, Holden-Day, San Francisco, 1971.

5. G. Hochschild and G. D. Mostow, Representations and representative functions of Lie groups, Ann. Math., 66 (1957), 495-542.

6. - On the algebra of representative functions of an analytic group, Amer. J. Math., 83 (1961), 111-136.

7. - On the algebra of representative functions of an analytic group, II, Amer. J. Math., 86 (1964), 869-887.

8. A. Magid, Analytic left algebraic groups, Amer. J. Math., 99 (1977), 1045-1059.

9. - Moduli for analytic left algebraic groups, Trans. Amer. Math. Soc., (to appear).

10. - Analytic subgroups of affine algebraic groups, Duke J. Math., 44 (1977), $875-882$.

Received April 3, 1978.

UNIVERSITY OF OKLAHOMA

Norman, OK 73019 


\section{PACIFIC JOURNAL OF MATHEMATICS}

\section{EDITORS}

DONALD BABBITT (Managing Editor)

University of Galifornia

Los Angeles, California 90024

HUGo RossI

University of Utah

Salt Lake City, UT 84112

C. C. MOORE AND ANDREW OGG

University of California

Berkeley, CA 94720
J. DUGUNDJI

Department of Mathematics University of Southern California Los Angeles, California 90007

R. FINN AND J. MILGRAM Stanford University Stanford, California 94305

\section{ASSOCIATE EDITORS}

E. F. BECKENBACH

B. H. NEUMANN

F. WOLF

K. YosHIDA

\section{SUPPORTING INSTITUTIONS}

UNIVERSITY OF BRITISH COLUMBIA CALIFORNIA INSTITUTE OF TECHNOLOGY UNIVERSITY OF CALIFORNIA MONTANA STATE UNIVERSITY UNIVERSITY OF NEVADA, RENO NEW MEXICO STATE UNIVERSITY OREGON STATE UNIVERSITY UNIVERSITY OF OREGON
UNIVERSITY OF SOUTHERN CALIFONIA STANFORD UNIVERSITY UNIVERSITY OF HAWAII UNIVERSITY OF TOKYO UNIVERSITY OF UTAH WASHINGTON STATE UNIVERSITY UNIVERSITY OF WASHINGTON 


\section{Pacific Journal of Mathematics}

\section{Vol. 86, No. $1 \quad$ November, 1980}

Gert Einar Torsten Almkvist, Invariants, mostly old ones .............. 1

Hyman Bass, Groups of integral representation type ................ 15

A. Białynicki-Birula, On action of SL(2) on complete algebraic

varieties........................................

Frederick Paul Greenleaf and Martin Allen Moskowitz, Groups of

automorphisms of Lie groups: density properties, bounded orbits, and

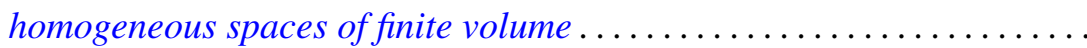

Raymond Taylor Hoobler, A cohomological interpretation of Brauer groups

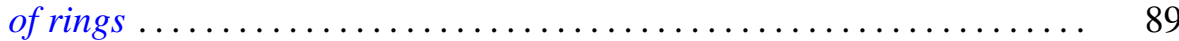

Irving Kaplansky, Superalgebras ........................ 93

Jerrold Lewis Kleinstein and Alex I. Rosenberg, Succinct and

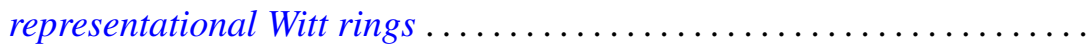

E. R. Kolchin, On universal extensions of differential fields ............ 139

Andy R. Magid, Analytic subgroups of affine algebraic groups. II ....... 145

Calvin Cooper Moore, The Mautner phenomenon for general unitary

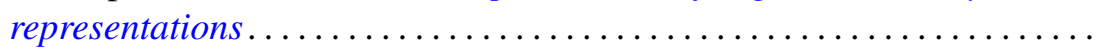

George Daniel Mostow, On a remarkable class of polyhedra in complex hyperbolic space ................................ 171

Brian Lee Peterson, Extensions of pro-affine algebraic groups. II . . . . . . 277

John Henry Reinoehl, Lie algebras and affine algebraic groups......... 287

Maxwell Alexander Rosenlicht, Differential valuations .

John Brendan Sullivan, The second Lie algebra cohomology group and Weyl modules..................................... 321

Moss Eisenberg Sweedler, Right derivations and right differential

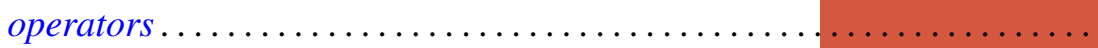

Bostwick Frampton Wyman, Time varying linear discrete-time systems. II. Duality.................................. 